\title{
Comparison of Post Operative Outcomes Following Del Nido Cardioplegia Versus Howards Cardioplegia in Adult Cardiac Surgery at Pakistan Institute of Medical Sciences
}

\author{
Adnan Shah, Attiya Hameed Khan *, Hammad Ahmed*, Aamir Naveed, Faridullah Khan, \\ Javeed Iqbal, Adnan Tahir
}

Department of Cardiac Surgery at Pakistan Institute of Medical Sciences, Bashir Institute of Health Sciences Affiliated with Shaheed Zulfiqar Ali Bhutto Medical University, Islamabad, Pakistan

\section{Email address:}

adnan.shah9930@gmail.com (A. Shah),Attiyahameed864@gmail.com (A. H. Khan), hammad.gakhar07@gmail.com (H. Ahmed), aamirnaveed@bashir.edu.pk ( $\Lambda$. Naveed),prof.faridullahkhan@gmail.com (F. Khan), Javedperfusionist@gmail.com (J. Iqbal), tgtg2@hotmail.com (A. Tahir)

${ }^{*}$ Corresponding author

\section{To cite this article:}

Adnan Shah, Attiya Hameed Khan, Hammad Ahmed, Aamir Naveed, Faridullah Khan, Javeed Iqbal, Adnan Tahir. Comparison of Post Operative Outcomes Following Del Nido Cardioplegia Versus Howards Cardioplegia in Adult Cardiac Surgery at Pakistan Institute of Medical Sciences. International Journal of Cardiovascular and Thoracic Surgery. Vol. 6, No. 1, 2020, pp. 1-6. doi: $10.11648 /$ j.ijcts.20200601.11

Received: November 19, 2019; Accepted: December 18, 2019; Published: February 4, 2020

\begin{abstract}
This study compares post-operative outcomes for ionotropic support, defibrillator need and duration of hospital stay in adult patients of cardiac surgery following Del Nido cardioplegia versus Howard's cardioplegia and also compare pre and post operative Cardiac enzymes and serum potassium levels, assessment of pre and post operative Arrthymias. It was an Observational study conducted at Pakistan Institute of Medical Sciences Islamabad Pakistan. Patients were divided into 2 groups. Group (A) patients were Del Nido cardioplegia and Group (B) patients were Howard's cardioplegia. Data was collected by using non-probability Consecutive Sampling technique. 100 patients were enrolled in this study randomly allocating 50 to each study group. Results of the study show that Post-op CKMB level after 24 hours for Howard group was

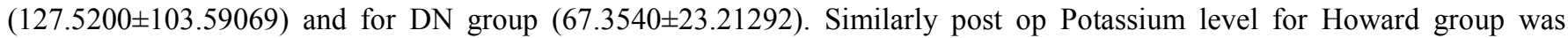

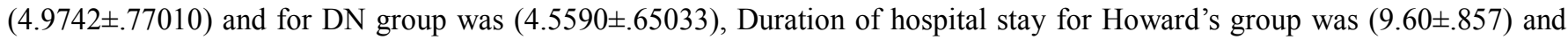
for DN group was $(8.58 \pm 1.486)$. High ionotropic support was required in Howard's group as compared to DN where it was mild to moderate. $40 \%$ patients required defibrillation in Howards as compared to DN with only $20 \%$. It is concluded that Cardioplegia is intended for brief cardiac arrest, which is an important function during cardiopulmonary Bypass surgery. After comparing two cardioplegic solutions namely Del Nido and Howard's cardioplegia. Our study concludes that Del-Nido cardioplegia provide better myocardial protection and less cross clamp and bypass time. Cardiac enzymes, serum potassium level and post-op arrthymias show better profile in Del-Nido as compared to Howard's cardiolpegia. Our data clearly advocate the efficacy and safety of Del-Nido over Howard's cardioplegia. It is very safe and effective as compared to Howards.
\end{abstract}

Keywords: Cardiopulmonary Bypass, Coronary Artery Disease, Adult Cardiac Surgery, Cardioplegia, Howards, Del-Nido, Cardiac Enzymes

\section{Introduction}

Coronary artery disease is the cause of approximately onethird of the world's population deaths in individuals over 35 years of age [1]. Globally over 800,000 coronary artery bypass grafts (CABG) surgery or valvular operations are reported per year, with around 1000 surgeries per day in the US alone. On an average In-hospital/30-day mortality rates are $1 \%$ for $\mathrm{CABG}$, for valve surgery it is $5-6 \%$ for valve surgery and $7 \%$ for combined CABG and valve surgery $[2,3]$.

Myocardial protection and functioning in this protocol is complicated by subsequent ischemia-reperfusion injury, 
intracellular $\mathrm{Ca}^{2+}$ overload and oxidative stress, all of which may contribute to post-operative myocardial dysfunction. Current cardioplegia options include whole blood cardioplegia (WB), warm blood cardioplegia and Buck berg solution [4].

Cardioplegia, one of the major protection techniques, has evolved in many different directions since the first report of elective cardiac arrest by Dr. Melrose in 1955 [5]. Ideal cardioplegia solution achieves rapid diastolic arrest and decreases myocardial metabolic demand to facilitate a quiescent heart amenable to surgical intervention. Upon reperfusion, injury to the myocardium should be minimized with swift return to non-ischemic sinus rhythm. Most of the Researchers developed a new formulation of Pittsburgh for myocardial protection at the University of Pittsburgh in early 1990s [7]. Modifications have been made to the original solution and it is now referred to as Del Nido cardioplegia in the literature and in clinical practice [8].

Del Nido cardioplegia have been reported to be related brief aortic cross clamp time and bypass time [6]. In 2016 study was done including patients with valvular heart disease requiring valve replacement and ischemic heart disease requiring single or multiple vessel bypass surgery, showed that cardiopulmonary bypass time, cross-clamp time and duration of hospital stay was same at $p$ value $>0.05,65 \pm 23$, $65 \pm 21$ minutes and $6.4 \pm 3,5.8 \pm 3$ days appropriately in both groups. Those patients who needs defibrillators was (42\%) and $(8 \%)$ at $(p<0.0001)$ [9]. In Recent surgeries mostly surgeon use Howards cardioplegia which was introduced in 1977 by Melrose and associates in 1955. It was his first effort by involving potassium concentration at the range of $245 \mathrm{mEq} / \mathrm{L}$, which cause damaging of myocardial layer. Mostly that type of injuries should be avoided through hypothermia and also through normothermia process [9]. Howard's cardioplegia were not used commonly after 1977. With the use of Howard's cardioplegia, physiology of blood found good and expandable in last 5 years.

There are many advantages of Howards cardioplegia if firstly disseminate and there is need to modify it. It cause rapid cardiac arrest with an oxygenated environment alternating to re-oxygenation delivery of cardioplegia which made it anaerobic substance with in blood, and provide oncotic complement and after that added as plasma protein, mannitol, or dextrin and there is a need for the avoidance of a complex pharmacological mixture and delivery apparatus which is more costly delivery system than for crystalloid cardioplegia [10].

Different cardioplegia techniques are being employed for safe cardiac surgery with minimum complications and quick recovery. Del Nido cardioplegia is considered to be safer technique now days with improved results when compared to previously frequently use Howard's cardioplegia solution which results in more prolonged cardioplegia and more complication rate. In the setting of Rawalpindi and Islamabad, mostly hospitals use Howard's cardioplegia solution because it is available in prepared form but some of them use Del Nido cardioplegia because of its time and dose amount. Some surgeon preferred to do cardiac surgery upon Howard's cardioplegia and most of them suggest to do work through Del Nido cardioplegia.

\subsection{Composition of Del-Nido Cardioplegia}

Composition of cardioplegia in every hospital is different, as in our setting the composition of Del Nido is: potassium chloride $(\mathrm{kcl}) 26 \mathrm{mEq}$, sodium bicarbonate (Na2HCO3) 13 $\mathrm{mEq}$, Magnesium (Mg), $2 \mathrm{~g}$, lignocaine $6.5 \mathrm{ml} / 130 \mathrm{mg} / \mathrm{dL}$, mannitol $16 \mathrm{ml}$, and the composition of Howards cardioplegia is: procaine hydrochloride $0.27 \mathrm{~g} / 49 \mathrm{~m} \mathrm{~mol}$, potassium chloride $(\mathrm{kcl}) 1.19 \mathrm{~g} / 16 \mathrm{~m} \mathrm{~mol}$, magnesium $(\mathrm{mg})$ $3.25 \mathrm{~g} / 16 \mathrm{~m}$ mol. Study was planned to compare different parameters between Del Nido cardioplegia and Howard's cardioplegia to assess both cardiologic solutions.

Therefore the objective of our study wasto compare postoperative outcomes for ionotropic support, defibrillator need and duration of hospital stay in adult patients undergoing cardiac surgery following Del Nido cardioplegia versus Howard's cardioplegia and Compare pre and post operative Cardiac enzyme (CKMB) and potassium levels in adult patients undergoing cardiac surgery and also detect Arrhymias in pre, post adult Cardiac surgery.

\subsection{List of Abbreviations}

There are few abbreviations in our whole paper, which are, BMI: Bashir Medical Institute, CPs: Cardioplegia Solution, CKMB: Creatine kinase-muscle/brain, Ca: Calcium, CPB: Cardiopulmonary Bypass, CAD: Coronary Artery disease, DN: Del-Nido, DF: Defibrillation, Mg: Magnesium, ICU: Intensive Care Unit, IP: Ionotropic Support, PIMS: Pakistan Institute of Medical sciences, LDH: Lactate dehydrogenate, SD: Standard Deviation, WHO: World health organization.

\section{Methodology}

This was an observational cross sectional study based on, to compare different parameters between Del Nido cardioplegia and Howard's cardioplegia to assess post operative outcomes of both cardioplegic solutions in adult Cardiac Surgery in Pakistan Institute of Medical Sciences. Study was conducted among the patients of adult cardiac surgery (CABG, single valve replacement or multiple valve replacement surgery and Elective surgery).

Ethical approval was taken from hospital ethics committee. Written formal informed consent was taken from patients. Study was conducted over a period of six months from November 2018 to April 2019. Sample size for the study was 100 patients were enrolled in the study randomly allocating 50 to each study group. Sample was collected by using nonprobability consecutive Sampling [7].

Age between 30-70 years, CABG, single valve replacement or multiple valve replacement surgery to be performed and Elective surgeries were included and Reoperation, Emergency operation, Left ventricular dysfunction $(\mathrm{EF}<30 \%$ ), Renal dysfunction (preoperative serum 
creatinine level $>1.5 \mathrm{mg} / \mathrm{dL}$ ) and cardiogenic shock were excluded from the study. After coding obtained filled
Performa's from patients data was entered and analyzed by using SPSS version 25.

\section{Results}

Table 1. Age, Gender and previous history of smoking for Del-Nido and Howard expressed as frequency and percentage of patient where $n=50$ each group.

\begin{tabular}{|c|c|c|c|c|}
\hline Characteristics & \multicolumn{2}{|c|}{ Howards $(n=50)$} & \multicolumn{2}{|c|}{ Del-Nido $(n=50)$} \\
\hline Age (years) mean $\pm \mathrm{SD}$ & \multicolumn{2}{|c|}{$50.42 \pm 10.389$} & \multicolumn{2}{|c|}{$50.92 \pm 9.426$} \\
\hline Gender & \multicolumn{2}{|c|}{ Frequency (Percentage) N, (\%) } & \multicolumn{2}{|c|}{ Frequency (Percentage) N, (\%) } \\
\hline Male & \multicolumn{2}{|c|}{$29(58.0)$} & \multicolumn{2}{|c|}{$42(84.0)$} \\
\hline Female & \multicolumn{2}{|c|}{$21(42.0)$} & \multicolumn{2}{|l|}{$8(16.0)$} \\
\hline Characteristics & \multicolumn{2}{|c|}{ Howards $(n=50)$} & \multicolumn{2}{|l|}{$\mathrm{DN}(\mathrm{n}=50)$} \\
\hline \multirow[t]{2}{*}{ Previous history of smoking } & Yes n $(\%)$ & NO n $(\%)$ & Yes n $(\%)$ & NO n $(\%)$ \\
\hline & $27(54.0)$ & $23(46.0)$ & $18(36.0)$ & $32(64.0)$ \\
\hline
\end{tabular}

Table 1 Illustrate the mean age of patients for Howards and del-Nido cardioplegia which were $50.42 \pm 10.389$ and $50.92 \pm 9.429$ and frequency and percentage of genders which shows that male and females for Howards were $29(58 \%)$ and $21(42 \%)$ and for del-Nido were $42(84 \%)$ and $8(16 \%)$, which shows that the percentage of males were higher in both group as compared to females. This table also shows the frequency and percentage of previous history of smoking of both group which shows that $27(54 \%)$ were smoker in Howards group and $18(36 \%)$ in del-Nido group.

Table 2. Comparison of pre and post operative outcomes of Howards and del-Nido group of patients, where $n=50$ each group.

\begin{tabular}{|c|c|c|c|c|c|c|c|}
\hline & Howard & & $\mathbf{D N}$ & & $95 \%$ CI & & \\
\hline & $\mathbf{n}=\mathbf{5 0}$ & & $\mathbf{n}=\mathbf{5 0}$ & & & & \\
\hline Scales & M & SD & M & SD & $\mathbf{t}$ & df & $\mathbf{p}$ \\
\hline Aortic cross clamp time & 50.78 & 6.685 & 46.24 & 16.561 & .007 & 98 & .004 \\
\hline By pass time $\mathrm{B}$ & $82 . .28$ & 9.927 & 79.86 & 22.924 & 1.973 & 98 & .004 \\
\hline CKMB post-op $>6$ hours & 81.8200 & 50.42824 & 58.2160 & 24.29223 & 2.982 & 98 & .004 \\
\hline CKMB post-op $>12$ hours & 127.5200 & 103.59069 & 67.3540 & 23.21292 & 4.008 & 98 & 004 \\
\hline LDH post-op $>6$ hours & 362.1380 & 75.05858 & 379.8800 & 119.23541 & .890 & 98 & .375 \\
\hline Potassium level Post-op & 4.9742 & .77010 & 4.5590 & .65033 & -2.913 & 98 & 004 \\
\hline Duration of hospital (Post-op) & 9.60 & .857 & 8.58 & 1.486 & -4.205 & 98 & .000 \\
\hline
\end{tabular}

Table 2 illustrate the Mean and standard deviations of pre and post outcomes of both groups of patients, which shows that Pre-op ejection fraction of Howards was 55.70 \pm 7.446 and for DN was 49.80 \pm 9.455 respectively ( $p<0.05$ ). Through observing Aortic cross clamp time in minutes for Howard's group was $50.78 \pm 6.685$ and for DN was $46.24 \pm 16.561$ respectively $(\mathrm{p}<0.05)$. Bypass time in minutes for DN group was significantly lower than Howards group, values being $79.86 \pm 22.924$ and $82.28 \pm 9$ respectively ( $p<$ $0.05)$. With DN cardioplegia significantly lower post op (after 6 and $24 \mathrm{hr}$ ) levels of CKMB was observed the values being (58.2160 \pm 24.29223$)(67.3540 \pm 23.2129)$ respectively as compared to Howard cardioplegia where it was $81.8200 \pm 50.42824$ and $127.5200 \pm 103.59069$ with $p<0.05$ by comparison of two groups.

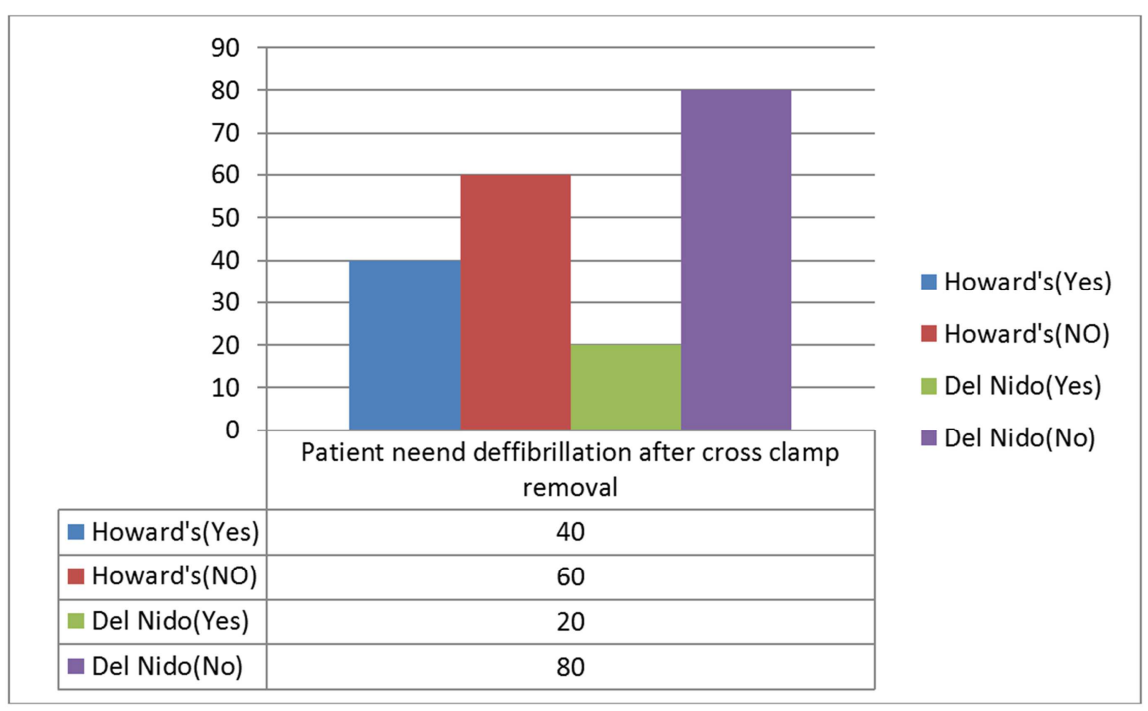

Figure 1. Defibrillation need after cross clamp removal in Del - Nido vs Howard group expressed as percentage of patient where $n=50$ each group. 


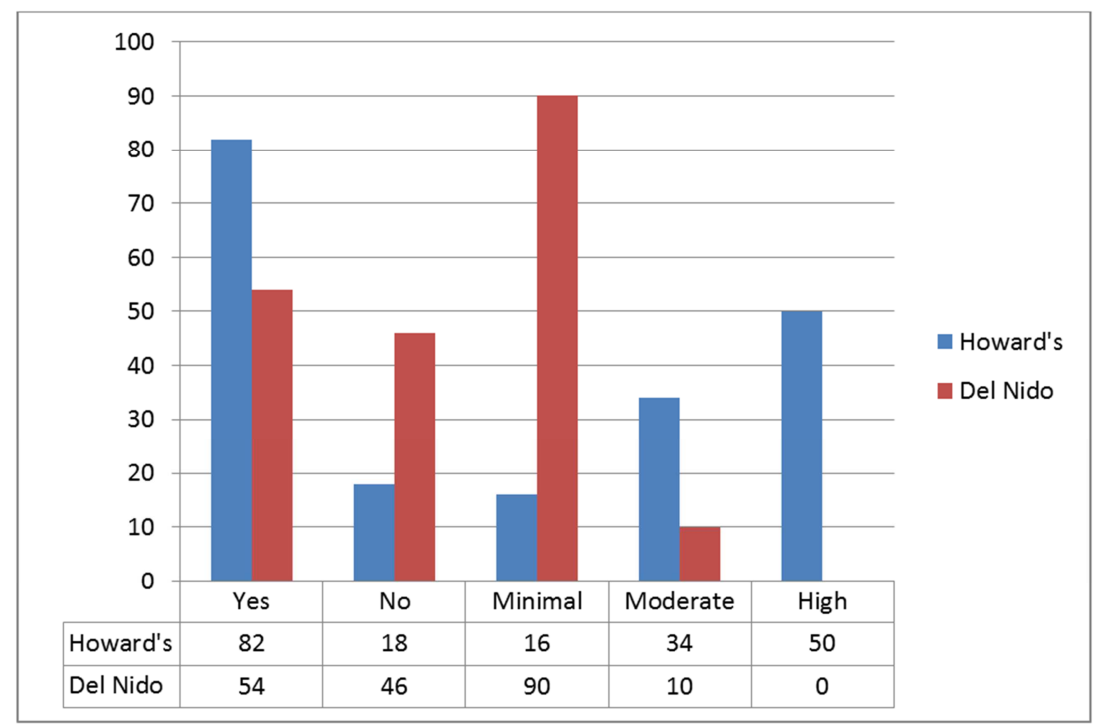

Figure 2. Ionotropic support in Dil-Nido vs. Howard group expressed as percentage of patient requiring (mild, modrate, high) where $n=50$ each group.

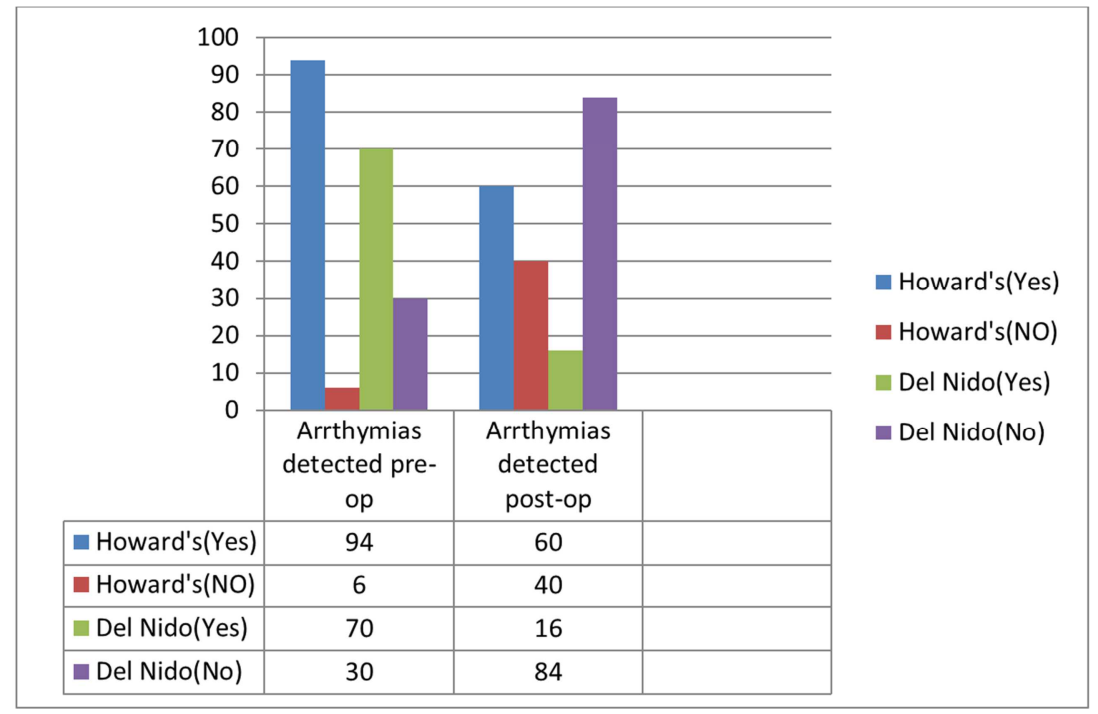

Figure 3. Arrhymias detection pre and post in Del-Nido versus Howards group expressed as percentage of patient where $n=50$ each group.

\section{Discussion}

Cardioplegia, one of the major protection techniques, has evolved in many different directions since the first report of elective cardiac arrest by Dr. Melrose in 1955 [5]. Ideal cardioplegia solution achieves rapid diastolic arrest and decreases myocardial metabolic demand to facilitate a quiescent heart amenable to surgical intervention. Upon reperfusion, injury to the myocardium should be minimized with swift return to non-ischemic sinus rhythm [6].

There are several components constituting Cardioplegia solutions. $\mathrm{Na}^{+}$ions serve the primary component for induction of cardiac arrest in extracellular space [10]. Secondary components are added to the cardioplegia solution for myocardial protection during cross clamp time, protection against metabolic stress and reperfusion injury during Cardiopulmonary Bypass surgery [11]. A previous study advocated the effectiveness and safety of Del-Nido cardioplegia containing modified lidgocaine, in adults with acquired heart diseases. DN cardioplegia when compared with conventional techniques delivered better post operative outcomes in terms of requirement of ionotropic support, ventilation time, development of AV block and mortality rate [12].

Our study suggests that number of patients requiring ionotropic support in Del-Nido was significantly lower than Howard's group (54\% DN, 82\% Howards). Similarly DN group patients need for post-op ionotropic support was either mild $(90 \%)$ and moderate $(10 \%)$, while in Howards group patients need ionotropic support was mild (16\%), Moderate (34\%) and high (50\%). These results clearly claim that DN provide best post-op outcome over Howards in term of frequency and intensity of post-op ionotropic support requirement.

According to the analysis of arrthymias in our study (pre \& post op) for Del-Nido, the number of patients with detected 
arrthymias was lower with pre-op (70\%) and post-op (16\%) while in Howard's group it was pre-op (94\%) and post-op $(60 \%)$ respectively. It shows that the percentage of Arrthymias detection in Del-Nido group were lower than Howards group.

As for as dosing frequency during cardiopulmonary bypass surgery is concerned, Del-Nido cardioplegia requires a single dose administration during whole procedure while for Howard's cardioplegia it requires repeated dose administrations. Through the use of Del-Nido cardioplegia, it was assessed that there was a shorter cross clamp time and bypass time table 2 . This can be attributed to the reduced requirement to administer repeated doses of cardioplegia. The cross clamp and bypass time in the present study were, however, shorter than those reported in previously published studies in adult patients [13].

\subsection{Effectiveness of Study}

Our data also shows that it is a single dose administration of Del-Nido solution provides better post-op outcomes when compared to multi-dose administration of Howards. It provides good myocardial protection and less detection of post-op arrthymias table 2 dosing variation in Howards was strongly associated with corresponding variations in detection of arrhythmias, need for defibrillation and ionic support table with $\mathrm{p}<0.05$. while, in case of Del-Nido no statistically strong association was found among dosing variations and respective alterations in arrthymias, defibrillation needs and ionotropic support $\mathrm{p}>0.05$ table 2 . This shows the better safety profile and wide therapeutic window of Del-Nido as compared to Howards.

\subsection{Limitations of the Study}

\subsubsection{Strength of the Study}

It was hospital base study and conducted by the validated Performa. Based upon the results of this study we promote the use of Del-Nido cardioplegia in terms of post operative outcomes as it provides an advantages over Howards cardioplegia, also we observed better myocardial protections after the use of Del-Nido cardioplegia when compared with Howards. This can serve a baseline for future research.

\subsubsection{Weakness of the Study}

It had limited sample size and only included the Howards and Del-Nido cardioplegia. It was consist of Post operative outcomes and not included the other measurement and also the other complications. It was consist of only Adult cardiac surgeries not included peadritic surgeries.

\section{Conclusion}

Cardioplegia is intended for brief cardiac arrest, which is an important function during cardiopulmonary Bypass surgery. After comparing two cardioplegic solutions namely Del Nido and Howard's cardioplegia. Our study concludes that Del-Nido cardioplegia provide better myocardial protection and less cross clamp and bypass time. Cardiac enzymes, serum potassium level and post-op arrthymias show better profile in Del-Nido as compared to Howard's cardiolpegia. Our data clearly advocate the efficacy and safety of Del-Nido over Howard's cardioplegia. It is very safe and effective as compared to Howards.

\section{Acknowledgements}

I pay my gratitude to my family, who stood by me and spared me for this uphill task. Their continuous support, prayers and sacrifices remained a source of inspiration for me in completion of my study.

I am indebted to my teachers, Madam Attiya Hameed khan, Dr Hammad Ahmed, Madam Syeda Anum Batool and Madam Sadia Noor who has always been very considerate and encouraging despite knowing my academic misdemeanors.

\section{References}

[1] Mangiacapra F., De Bruyne B., Wijns W., Bartunek J. Optimizing revascularization strategies in coronary artery disease for optimal benefit to patients. Clin. Pharmacol. Ther. 2011.

[2] Hannan EL, Racz M, Culliford AT, Lahey SJ, Wechsler A, Jordan D, et al. Risk Score for predicting in-hospital/30-day mortality for patients undergoing valve and valve/coronary artery bypass graft surgery. Ann. Thorac. Surg. 2013.

[3] Ben-Gal Y, Stone GW, Smith CR, Williams MR, Weisz G, Stewart AS, et al. On-pump versus off-pump surgical revascularization in patients with acute coronary syndromes: analysis from the Acute Catheterization and Urgent Intervention Triage Strategy trial. J Thorac Cardiovasc Surg. 2011.

[4] Yerebakan H, Sorabella RA, Najjar M, Castillero E, Mongero L, Beck J, et al. Del Nido Cardioplegia can be safely administered in high-risk coronary artery bypass grafting surgery after acute myocardial infarction: a propensity matched comparison. Journal of Cardiothoracic Surgery 2014.

[5] Richard M. Ginther, Jr., CCP; Ronald Gorney, PA-C, CCP; Joseph M. Forbess, Use of del NidoCardioplegia Solution and a Low-Prime RecirculatingCardioplegia Circuit in Pediatrics. JECT. 2013.

[6] Loberman D, Neely RC, Fitzgerald D, McGurk S, Rajab TK. Modified Del Nido Cardioplegia in Adult Cardiac Surgery; Safety and Efficacy. J Cardiol CurrRes. 2014.

[7] Marill KA, Salcido DD, Sundermann ML, Koller AC, Menegazzi JJ. Potassium cardioplegia during CPR for porcine VF arrest: A blinded randomized controlled trial. 2015.

[8] Guajardo Salinas GE, Nutt R, Rodriguez-Araujo G. Del Nido cardioplegia in low risk adults undergoing first time coronary artery bypass surgery. Perfusion 2016.

[9] Lawton JS. 50th Anniversary Landmark Commentary on Gay WA. Potassium-induced cardioplegia. Ann Thorac Surg 1975; 20: 95-100. Ann Thorac Surg 2015. 
[10] Merbel N, Savoie N, Yadav $M$, et al. Stability: recommendation for best practices and harmonization from the Global Bioanalysis Consortium Harmonization Team. AAPS J. 2014.

[11] Matte GS, del Nido PJ. History and use of del Nido cardioplegia solution at Boston Children's Hospital. JECT 2012.

[12] Vistarini N, Laliberté E, Beauchamp P, Bouhout I, Lamarche Y, Cartier R, Carrier M, Perrault L, Bouchard D, El-Hamamsy I, Pellerin M, Demers P. Del Nido cardioplegia in the setting of minimally invasive aortic valve surgery. Perfusion. 2017.

[13] Guajardo Salinas GE, Nutt R, Rodriguez-Araujo G. Del Nido cardioplegia in low risk adults un). Charette K, Gerrah R,
Quaegebeur J, et al: Single dose myocardial protection technique utilizing del Nido cardioplegia solution during congenital heart surgery procedures. Perfusion 2012.

[14] Govindapillai A, Hua R, Rose R, et al. Protecting the aged heart during cardiac surgery: use of del Nido cardioplegia provides superior functional recovery in isolated hearts. $\mathrm{J}$ Thorac Cardiovas Surg 2013.

[15] Smigla G, Jaquiss R, Walczak R, et al. Assessing the safety of del Nidocardioplegia solution in adult congenital cases. Perfusion 2014.

[16] Sanetra K, Pawlak I, Cisowski M. Del Nido cardioplegia - what is the current evidence?. Kardiochir Torakochirurgia Pol. 2018. 